\title{
Adenocarcinoma at the gastroesophageal junction
}

\author{
Sahar Al-Haddad, ${ }^{1}$ Andrew C. Chang, ${ }^{2}$ Gert De Hertogh, ${ }^{3}$ Andrea Grin, ${ }^{4}$ Rupert Langer, ${ }^{5}$ \\ Xavier Sagaert, ${ }^{3}$ Marianna Salemme, ${ }^{6}$ Catherine J. Streutker, ${ }^{1}$ Geneviève Soucy, ${ }^{7}$ \\ Monika Tripathi, ${ }^{8}$ Melissa P. Upton, ${ }^{9}$ Michael Vieth, ${ }^{10}$ and Vincenzo Villanacci ${ }^{6}$ \\ ${ }^{1}$ Department of Laboratory Medicine and Pathobiology, St. Michael's Hospital, Toronto, Canada. ${ }^{2}$ Section of Thoracic Surgery, \\ University of Michigan Medical Center, Ann Arbor, Michigan. ${ }^{3}$ Department of Morphology and Molecular Pathology, University \\ Hospitals of K.U. Leuven, Leuven, Belgium. ${ }^{4}$ St. Michael's Hospital, Toronto, Canada. ${ }^{5}$ Institute of Pathology, University of \\ Bern, Bern, Switzerland. ${ }^{6}$ Department of Pathology, Spedali Civili, Brescia, Italy. ${ }^{7}$ Département de Pathologie - Pathologie \\ Gastro-intestinale, Centre Hospitalier de I'Université de Montréal, Montréal, Canada. ${ }^{8}$ Department of Cellular Pathology, \\ Oxford University Hospitals NHS Trust, Oxford, United Kingdom. ${ }^{9}$ Department of Pathology, University of Washington, Seattle, \\ Washington. ${ }^{10}$ Institute of Pathology, Klinikum Bayreuth, Bayreuth, Germany
}

Address for correspondence: annals@nyas.org

The following, from the 12th OESO World Conference: Cancers of the Esophagus, includes commentaries on the clinical differences between carcinomas arising slightly above, slightly below, and within the gastroesophageal junction (GEJ); information provided by biopsies; information provided by resection specimens following neoadjuvant therapy; histologic differences existing between carcinomas arising slightly above, slightly below, and within the GEJ; differences provided by immunohistochemistry in these tumors; information given by endoscopic mucosal resection specimens; the role of esophageal pyloric gland adenomas as precursors of adenocarcinomas in the region of the cardia; the role of pancreatic metaplasia; Her2 immunoreactivity to make distinctions in the site of origin; and intestinal metaplasia limited to the cardia as a precursor of adenocarcinoma.

Keywords: Her2; gastroesophageal junction; neoadjuvant; immunohistochemistry; pyloric gland adenoma; intestinal metaplasia; OESO

\section{Concise summary}

Clinical distinctions tend to segregate type I adenocarcinoma of the gastroesophageal junction (GEJ) from types II and III. Oncologically, patients with type III GEJ carcinomas tend to present at later stages and appear to have worse survival following resection than those with type I and type II. Oncologically, patients with type III carcinomas tend to present at later stages and appear to have worse survival following resection than patients with type I and type II, although this may be more dependent on tumor stage at presentation than anatomic location. When examining patterns of lymph node metastases, patients with type I were found to have involvement of posterior mediastinal paraesophageal lymph nodes in addition to upper abdominal lymph node basins. The staging systems for esophageal and gastric carcinomas have become more uniform and translatable, particularly when considering GEJ carcinomas.

The tumor morphology does not help to distinguish the distal esophageal adenocarcinoma (EAC) from the adenocarcinoma of the GEJ or proximal stomach, especially when it is of intestinal subtype. On biopsies, the tissue available for interpretation is often limited, as sampling is usually limited to the tumor and distal stomach mucosa is not available. Reported features helpful in distinguishing distal esophageal from proximal gastric columnar mucosa are the presence of severe crypt atrophy and disarray, especially if they are combined with findings of diffuse incomplete intestinal metaplasia (IM); squamous epithelium overlying crypts, multilayered epithelium, esophageal glands and/or ducts; and presence of hybrid glands. In biopsies of the GEJ region, although there are certain histologic features that can help pathologists determine whether a 
particular biopsy with columnar epithelium was obtained from the distal esophagus or the proximal stomach, these features are only present in about $30 \%$ of cases.

For tumors that show a high degree of regression following neoadjuvant therapy, one may only be able to identify a scarred tumor bed with superficial ulceration or even re-epithelization. The rates of significant tumor regression are higher in EACs type I as compared to adenocarcinomas of the GEJ (types II and III). Likewise, the probability for the latter tumors to achieve tumor regression is higher than in more distally located gastric cancers. These findings may be helpful for therapeutic decisions before initiating preoperative therapy in terms of response prediction. According to the current definitions of the World Health Organization (WHO) and the Union Internationale Contré le Cancer/American Joint Committee on Cancer (UICC/AJCC), the presence of Barrett's mucosa does not refine EAC from adenocarcinoma of the GEJ, since anatomic landmarks and not molecular genetic origin are considered as crucial for the classification of adenocarcinomas arising in this area.

No studies formally comparing adenocarcinomas slightly above, at, and below the GEJ have been published, investigating the histological differences between intestinal-type mucosa-associated and cardiac-type mucosa-associated adenocarcinomas. However, recent studies showed that EACs are more frequently well differentiated and gland forming than gastric cancers. In addition, the comparison of surgically resected EACs (Siewert type 1) with GEJ adenocarcinomas (Siewert type II) showed that all tumor types were present in both locations, but tumors associated with IM in the esophagus showed glandular morphology significantly more often.

There are trends of immunohistochemical differentiation by location in the phenotype of adenocarcinoma; however, that phenotype is not specific for location. The human embryonic esophagus is columnar lined in early development and undergoes squamous epithelialization in utero by a series of changes in gene expression. Therefore, immunohistochemistry is more sensitive and specific for patterns of differentiation or gene expression than for anatomical location. Barrett's neoplastic differentiation may be in an intestinal (goblet cell metaplastic) or a gastric foveolar direction, the latter without goblet cell phenotype. Both foveolar dysplasia in the esophagus and gastric adenocarcinomas share similar staining patterns, including loss or aberrant expression of lethal giant larva 2 (LGL2) in foveolar dysplasia, compared to reactive gastric mucosa. In contrast, expression of LGL2 is also frequently lost in areas of IM. Although the majority of esophageal and GEJ adenocarcinomas display intestinal-like immunophenotype, cardiactype differentiation is definitely not limited to or specific for adenocarcinomas arising in the GEJ or stomach, as gastric foveolar dysplasia can be seen in a significant subset of EACs. Finding Heliobacter pylori organisms by immunohistochemistry suggests an origin of the adenocarcinoma in the stomach, below the GEJ, rather than a GEJ or distal esophageal origin.

Regardless of whether a small amount of mucous or mixed mucous/oxyntic glands (i.e., cardia or cardiac-type mucosa) at the GEJ is normal, evidence shows that its length increases with age, acid exposure, and with histologic evidence of esophagitis and carditis. Therefore, metaplastic esophageal mucosa is essentially similar to the histology of cardiac mucosa and it can be very difficult, if not impossible, to distinguish these histologically in some cases. Endoscopic mucosal resection (EMR) specimens ideally include submucosal tissue and are helpful in assessing esophageal location through identification of features such as submucosal glands/ducts, duplicated muscularis mucosae, squamous islands/buried columnar mucosa, and palisade vessels.

To study the possible neoplastic potential of pancreatic acinar metaplasia (PAM) at the GEJ, the expression of c-erbB2, a receptor tyrosine kinase implicated in the development of pancreatic, esophageal, and gastric cancers, and K-ras, a tumor oncogene expressed in over $80 \%$ of pancreatic adenocarcinomas, was studied in patients with PAM with no evidence of BE. Neither gene was expressed.

A relationship between PAM at the GEJ and gastroesophageal reflux disease (GERD) and $H$. pylori infection in the stomach has been shown, with a potential neoplastic potential. The possible expression of c-erbB2, a receptor tyrosine kinase implicated in the development of pancreatic, esophageal, and gastric cancers, and K-ras, a tumor oncogene expressed in over $80 \%$ of pancreatic adenocarcinomas, was studied in patients with PAM with no evidence of $\mathrm{BE}$, but neither was expressed. 
In several studies, positivity for Her2, a transmembrane receptor protein belonging to the epidermal growth factor receptor (EGFR) family, was related to aggressive tumors and poor outcome. It has been shown that Her2 positivity is strongly associated with the intestinal path of tumor progression at the GEJ, rather than the cardiac type/foveolar dysplasia. In contrast, there were no significant differences between the groups in pathology or outcome. The differing rates of Her2 expression between the esophagus, GEJ, and distal stomach may all be due to the variation in the rates of intestinal-type cancers between the sites.

\section{Are there clinical features that distinguish between adenocarcinomas arising slightly above, slightly below, or at the GEJ?}

\author{
Andrew C. Chang \\ andrwchg@umich.edu
}

The GEJ, also designated the gastric cardia, indicates the point of transition from esophageal squamous epithelium to gastric mucosa. It has become widely accepted that a malignancy, typically an adenocarcinoma, with its epicenter arising within $5 \mathrm{~cm}$ proximal or distal to this site, can be classified by the anatomic-topographic classification described by Siewert et al.: ${ }^{1}$ type I tumors develop proximal to the cardia and infiltrate the GEJ distally; type II tumors are centered at the cardia; type III tumors arise within $5 \mathrm{~cm}$ distal to the GEJ and extend proximally. While the GEJ is defined by endoscopic and histologic criteria, the squamocolumnar junction or Z-line, often used as a reference point defining the cardia, can be difficult to identify clinically, particularly in the setting of extensive IM. Clinical distinctions tend to segregate type I adenocarcinoma of the GEJ (AEG) from types II and III AEG. Male sex, history of GERD, presence of a hiatal hernia, and identification of IM of the distal esophagus (Barrett's esophagus (BE)) are factors more commonly associated with type I AEG than the more distal types II and III tumors. ${ }^{2,3}$

Oncologically, patients with type III carcinomas tend to present at later stages and appear to have worse survival following resection than patients with type I and type II GEJ carcinomas, ${ }^{4}$ although
IM limited to the cardia is a particular histological finding that is detected with variable frequency by different series, depending on several elements, including endoscopic techniques, location/number of biopsies, and histological methods. Most authors identified $H$. pylori infection and reflux disease as the main factors in the onset of cardiac inflammation and hence of intestinal metaplasia, emphasizing the role of IM as risk factor for cancer development. Intestinal metaplasia is a frequent finding in the cardiac region, even when endoscopy is normal and has an important significance in the progression toward dysplasia and cancer.

this may be more dependent on tumor stage at presentation than anatomic location. Pathologic staging reported in several contemporary surgical series indicated that patients referred for resection of more distal AEGs (i.e., types II or III) were more likely to have increasingly advanced stage of disease with higher grade tumors compared to proximal AEGs. ${ }^{4,5}$ The majority of patients undergoing resection for adenocarcinomas of the GEJ were found to have lymph node involvement, ${ }^{4-6}$ with a tendency for more extensive lymph node involvement observed in the distal type III AEGs. ${ }^{4,5}$

When examining patterns of lymph node metastases, patients with type I AEGs were found to have involvement of posterior mediastinal paraesophageal lymph nodes in addition to upper abdominal lymph node basins. The more distal type II and III AEGs had markedly less involvement of mediastinal lymph nodes, with disease residing predominantly in the upper abdominal lymph node basins, notably the paracardial, lesser curvature, celiac axis, and greater curvature regions. ${ }^{4,6,7}$ With the adoption of the seventh edition of the AJCC and UICC staging criteria for esophageal cancer, arising from the Worldwide Esophageal Cancer Collaboration, ${ }^{8}$ the staging systems for esophageal and gastric carcinomas have become more uniform and translatable, particularly when considering GEJ carcinomas. ${ }^{9,10}$ As the esophagus lacks a serosa, tumor ( $\mathrm{T}$ ) staging continues to differ in terms of transmural invasion, in which $\mathrm{T} 3$ gastric cancers are defined as invading the serosa, whereas T3 esophageal cancers extend to the adventitia. In the seventh edition AJCC/UICC TNM staging schema, 
however, criteria for lymph node $(\mathrm{N})$ staging of esophageal carcinomas focuses primarily on the number of suspected or pathologically confirmed metastatic lymph nodes. Anatomic location (e.g., celiac axis lymph node involvement previously considered as metastatic disease (M1a)) is no longer considered in the nodal $(\mathrm{N})$ staging schema.

\section{How does the pathologist tell from biopsies?}

Geneviève Soucy

genevieve.soucy.chum@ssss.gouv.qc.ca

When evaluating biopsies of the GEJ region, knowledge of the exact biopsy site and endoscopic impression is essential. Usually, pathologists rely on endoscopists for conveying information regarding the anatomic site of biopsy samples. Determining the precise site of the biopsy may be difficult for the endoscopist, especially when the tumor obliterated the anatomic landmarks.

Answering this question is thus very challenging for pathologists for several reasons. First, the tumor morphology does not help to distinguish distal EAC from adenocarcinoma of the GEJ or proximal stomach, especially when it is of intestinal subtype. Esophageal and gastric adenocarcinomas of intestinal subtype are histologically indistinguishable, having arisen on a background of IM. ${ }^{11,12}$ Second, on biopsies, the tissue available for interpretation is often limited. Indeed, sampling is usually limited to the tumor, with the objective of establishing a diagnosis of malignancy. Often, sampling of adjacent mucosa or distal stomach mucosa is not available.

Based on the recognized etiologic mechanisms that EAC is strongly associated with GERD and $\mathrm{BE}$ with healthy gastric mucosa, and gastric adenocarcinoma is strongly associated with $H$. pylori gastritis, gastric atrophy, and IM, pathologists can look for the presence of nontumoral mucosa to help them identify the probable site of origin, as either esophageal or gastric. ${ }^{12-15}$

Useful nontumoral mucosa includes adjacent or distal gastric or esophageal mucosa. Despite the finding of dysplasia in these biopsies, other features have been reported to be helpful in distinguishing distal esophageal from proximal gastric columnar mucosa.

In biopsies of the GEJ region, differentiation of metaplastic esophageal columnar epithelium from normal proximal stomach (cardia), regardless of the presence or absence of goblet cells, is often difficult or impossible. However, few histologic features have been reported to help locate the mucosa at the GEJ in a setting of GERD. ${ }^{16}$

Reported features helpful in distinguishing distal esophageal from proximal gastric columnar mucosa are the presence of severe crypt atrophy and disarray; especially if they are combined with findings of diffuse incomplete IM, squamous epithelium overlying crypts (buried columnar epithelium), multilayered epithelium, esophageal glands and/or ducts, and presence of hybrid glands. Unfortunately, the presence of goblet cells, by itself, does not allow the pathologist to determine if a biopsy is esophageal or gastric in location. ${ }^{16}$ The coexistence of more than four of these features was $95 \%$ sensitive and $95 \%$ specific for predicting diagnosis of $\mathrm{BE}$ in the setting of GERD, known to be associated with EAC. ${ }^{16}$

In biopsies of the GEJ region, although there are certain histologic features that can help pathologists determine whether a particular biopsy with columnar epithelium was obtained from the distal esophagus or the proximal stomach, these features are only present in about $30 \%$ of cases. ${ }^{16}$

Biopsy samples from native esophageal epithelium and distal stomach should be obtained during the same procedure. Indeed, these biopsies are quite useful, since most $H$. pylori-associated carditis cases also show $H$. pylori antritis, thus favoring an adenocarcinoma of gastric origin. In contrast, active reflux esophagitis combined with the finding of a normal antrum or corpus are strong evidence in favor of GERD and adenocarcinoma of esophageal origin. ${ }^{12,15,17-19}$

In conclusion, pathologists can help endoscopists in identifying the site of origin of an adenocarcinoma based on recognized etiologic mechanisms, as long as adjacent mucosa or distal stomach mucosa samples are available for interpretation. Even with those biopsy samples, a minority of cases show helpful features and could possibly be correctly classified according to their site of origin.

\section{How does the pathologist tell from resection specimens following neoadjuvant therapy?}

\author{
Rupert Langer \\ rupert.langer@pathology.unibe.ch
}



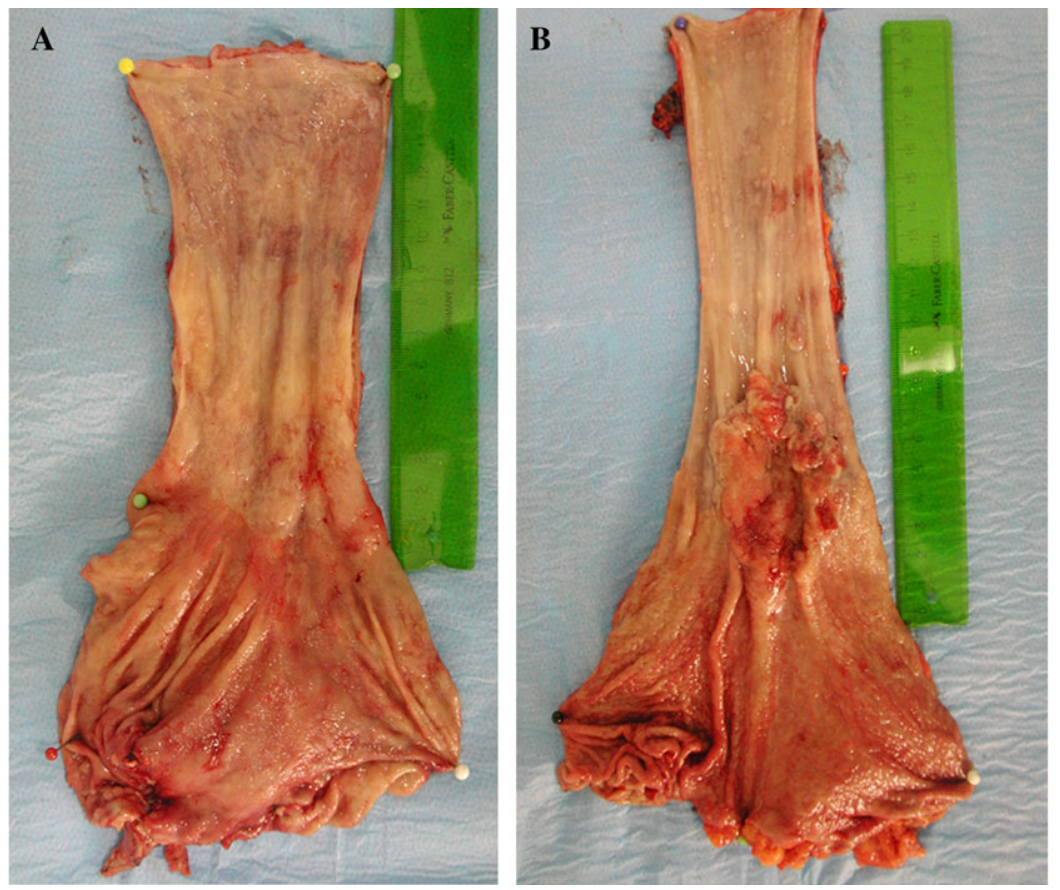

Figure 1. Macroscopic examples of esophagectomy resection specimens with adenocarcinomas of the esophagus/GEJ after neoadjuvant chemotherapy with (A) significant regression and (B) no regression. It is hard to appreciate the original site of the tumor in A since there now is only a scarred tumor bed visible. The tumor probably was located within the GEJ. In contrast, in B the tumor appears quite unchanged after treatment, and one would classify this tumor as esophageal cancer just not reaching the GEJ.

Gastroesophageal adenocarcinomas show a considerable variability in response to neoadjuvant treatment. ${ }^{20-22}$ In cases of no or little regression, the macroscopic appearance of the tumor may reflect the pretherapeutic conditions. In these cases, the appreciation of the origin of the tumor and a classification into one of the three sites of origin (esophagus, GEJ, stomach; or following the Siewert classification: AEG I, II, III or stomach) may be possible. In contrast, for tumors that show a higher degree of regression, one may only be able to identify a scarred tumor bed with superficial ulceration or even re-epithelization (Fig. 1). Under these circumstances, it can be impossible to precisely determine the localization of the initial tumor on the basis of the macroscopic or even histologic appearance of the resection specimen.

However, in the management of locally advanced gastroesophageal adenocarcinomas, which currently consists of neoadjuvant or perioperative treatment followed by surgery, most of the diagnostically and therapeutically relevant decisions have already been made before start of the preoperative therapy.
The presence of the tumor is diagnosed based on the endoscopic picture, but there is also the diagnostic biopsy taken from the tumor during endoscopy. In many cases, the determination of the tumor site is also done based on the relation of the tumor to landmarks such as the Z-line or the gastric folds within this investigation. Endo-ultrasound and other imaging diagnostics confirm the local or disseminated extension of the tumor. The results of these early investigations have immediate influence on further management, such as the decision in favor or against multimodal treatment concepts. In addition, the subsequent surgical approach will also be determined based on this categorization.

Interestingly, the rates of significant tumor regression are higher in EACs/AEG I as compared to adenocarcinomas of the GEJ/AEG II and III. ${ }^{22,23}$ Likewise, the probability for the latter tumors to achieve tumor regression is higher than in more distally located gastric cancers. ${ }^{23}$ These findings may be helpful for therapeutic decisions before initiating preoperative therapy in terms of response prediction. There is the general observation that patients with completely regressed tumors have a very 
good clinical outcome. Tumors without significant regression have a far worse prognosis. For tumors with partial or subtotal regression, there may exist differences regarding the effects of the degree of regression. Several studies show that patients with these tumors (e.g., 1-50\% residual tumor) show an intermediate prognosis between complete responders and nonresponders. ${ }^{21,22}$ In contrast, adenocarcinomas of the GEJ/AEG II and III are more similar to gastric cancer, where carcinomas with total and subtotal regression (e.g., $<10 \%$ residual tumor) have been shown to have a significantly better prognosis compared to partial or no regression (i.e., $>10 \%$ residual tumor). ${ }^{20}$ The classification of tumor regression, however, is not internationally or even nationally standardized. ${ }^{24} \mathrm{~A}$ more detailed discussion about the various tumor regression-grading systems would be far beyond the borders of this report.

At last, an interesting finding has been reported by Theisen et al., who describe the phenomenon of unmasking Barrett's mucosa through neoadjuvant chemotherapy. ${ }^{25}$ In these cases, an origin as an EAC arising within a Barrett's mucosa can be postulated. However, according to the current definitions of the $\mathrm{WHO}^{26}$ and the UICC/AJCC, ${ }^{27,28}$ the presence of Barrett's mucosa does not refine EAC from adenocarcinoma of the GEJ, since anatomic landmarks and not molecular genetic origin are considered as crucial for the classification of adenocarcinomas arising in this area.

\section{Adenocarcinomas slightly above, slightly below, and within the esophagogastric junction: are they histologically different?}

\section{Xavier Sagaert and Gert De Hertogh \\ xavier.sagaert@uzleuven.be}

Adenocarcinomas of the distal esophagus, GEJ, and cardia continue to rise in incidence, especially in the Western world. An intestinal metaplasia (BE)dysplasia-carcinoma sequence induced by GERD has been well established. Barrett's metaplasia is a predominant risk factor for the development of adenocarcinoma (30- to 50-fold increased risk in comparison to the general public). However, a significant number of adenocarcinomas in the vicinity of the GEJ are seen in the background of gastric/cardiac-type mucosa without IM. This has led to a couple of studies investigating, among other parameters, the histological differences between intestinal-type mucosa-associated and cardiac-type mucosa-associated adenocarcinomas.

Older studies demonstrated the presence of some clinicopathological differences between esophageal, cardiac, and distal gastric cancers. In 1984, Kalish et al. found that 23 esophageal and 49 cardiac adenocarcinomas (all surgically resected) were almost identical with regard to growth pattern (expansile vs. infiltrative), tumor grade, and tumor stage. ${ }^{29}$ This was more or less confirmed by Wang et al. in 1986, when comparing 12 esophageal, 9 GEJ, and 26 surgically resected cardiac adenocarcinomas; while no significant differences were found between esophageal and cardiac adenocarcinomas in terms of tumor grade, they did biologically behave differently from infracardial gastric adenocarcinomas: the presence of signet ring cells was less frequently observed, and signet ring cell differentiation was less extensive when present. $^{22}$ In 1993, Heidl et al. came to the same conclusion by comparing a large series of 66 esophageal, 359 cardiac, and 1288 infracardial adenocarcinomas: no significant differences between esophageal and cardiac adenocarcinomas could be found for Borrmann's, WHO, Ming's, and Laurén's classifications. ${ }^{30}$

Two more recent studies showed that EACs are more frequently well differentiated and gland forming than gastric cancers. In 2006, 215 esophageal, 108 cardiac, and 184 gastric adenocarcinomas (tumor location defined by the Siewert criteria) were histologically graded, staged, and typed according to the Carneiro's, WHO, and Laurén's classifications by Sarbia. ${ }^{31}$ It was found that esophageal cancers displayed the same histological spectrum as cardiac and gastric cancer; however, the relative proportion of differentiated, gland-forming carcinomas was significantly more frequent in the esophagus than in the cardia or in the stomach. Remarkably, in this particular study, among EACs, Lauren's classification, Carneiro's classification, tumor grade, and lymphatic vessel invasion, but not the WHO classification, were histological predictors of postoperative survival. In 2011, Demicco et al. clinicopathologically compared 51 surgically resected EACs (Siewert type I) with $106 \mathrm{GEJ}$ adenocarcinomas (Siewert type II). ${ }^{14}$ It was found that all tumor types (low- and high-grade glandular type, signet ring cell, mucinous, medullary, adenosquamous) were present in 
both locations, but tumors associated with intestinal (Barrett's) metaplasia in the esophagus showed glandular morphology significantly more often (even after correcting for tumor diameter).

Despite the fact that the results arising from older and more recent studies investigating the histological features in esophageal, GEJ, and cardiac adenocarcinomas are somewhat conflicting, the number of studies analyzing the histological differences remains limited, and so far, no studies formally comparing adenocarcinomas slightly above, at, and below the GEJ have been published.

\section{Are there immunohistochemical differences between carcinomas arising slightly above, slightly below, or at the GEJ?}

\section{Melissa P. Upton \\ mupton@uw.edu}

If immunohistochemical differences are detected, are they dependent on anatomic site or on embryologic origin? Do they reflect differences in etiology, such as $H$. pylori versus reflux? Are they markers of differentiation, such as intestinal versus gastric foveolar differentiation, rather than location?

First, how is location defined? Most studies use the Siewert classification, which defines type I as having an epicenter $1-5 \mathrm{~cm}$ proximal to the GEJ; type II with epicenter $2-5 \mathrm{~cm}$ distal to the GEJ, (subcardial, invading from below); and type III with epicenter located between $1 \mathrm{~cm}$ proximal and $2 \mathrm{~cm}$ distal to the GEJ (true GEJ tumors). ${ }^{2}$ Although the location can be defined after esophagogastrectomy, the preoperative endoscopic localization of the GEJ remains problematic. In one study, gastroenterologists $(n=80)$ were asked to identify the proximal gastric folds from photomicrographs of the GEJ taken from 30 patients with BE. The $\kappa$ coefficient for identification of the proximal gastric folds was only 0.35 , even after training. ${ }^{32}$ It is unlikely that in vivo endoscopic GEJ identification could be more reliable; hence, site designation of endoscopic biopsies is not precise, and studies based on endoscopic location are confounded by this limitation.

How is location above, below, or at the GEJ related to embryological origin? In the early embryo, the stomodeum gives rise to the foregut. Structures that arise from the foregut include the esophagus, stomach, duodenum, liver, pancreas, and respiratory system. As foregut derivatives, they may share certain immunohistochemical markers. This explains the fact that carcinomas arising in this broad region can express markers associated with differentiation into other foregut-derived tissues. Thus, we can find EACs that have Hepar-1 cytoplasmic expression, TTF-1 nuclear expression, and/or CDX-2 expression. The human embryonic esophagus is columnar lined in early development and undergoes squamous epithelialization in utero through a series of changes in gene expression. Therefore, immunohistochemistry is more sensitive and specific for patterns of differentiation or gene expression than for anatomical location. And subtle differences in location over the $10-\mathrm{cm}$ region that spans the GEJ are unlikely to result in specific immunohistochemical differences among neoplasms related to whether they arise above or below the GEJ.

Animal models provide additional evidence that the GEJ is a transition zone populated by migrating cell populations, depending on physiological setting and gene expression. The $p 63$ knockout mouse model illustrates the role of migratory cell populations to GEJ epithelialization and possible neoplasia. In stratified squamous epithelia, p63 is required for stem cell regeneration, but it is not required for lineage differentiation in nonsquamous directions. In the $p 63$ knockout mouse model, the number of p63expressing squamous stem cells is reduced. If the animals are subjected to acid reflux that damages the distal esophagus, residual embryonic cells of gastric origin migrate into the distal esophagus, leading to replacement of the squamous lining by columnar lining of gastric type (i.e., columnar metaplasia). Similarly, altered expression of p63 in humans might lead to incomplete squamous epithelialization during fetal life or aberrant re-epithelialization after injury, such as acid reflux..$^{14,33,34}$

Barrett's neoplastic differentiation may also be in an intestinal (goblet cell metaplastic) or a gastric foveolar direction, the latter without goblet cell phenotype. In a Cleveland Clinic study of 3698 biopsies with BE, 1779 biopsies from 461 patients had dysplasia. One hundred and sixty (8.9\%) of the biopsies from 44 (9.5\%) patients had foveolar dysplasia only, including 74 low-grade and 86 high-grade biopsies, and these were investigated immunohistochemically. In this study, MUC2 
Table 1. Immunohistochemical characteristics of neoplasms, compared according to their background mucosa

\begin{tabular}{ll}
\hline Intestinal-type background & Cardiac-type background \\
\hline $\begin{array}{l}\text { More commonly low grade with nuclear } \beta \text {-catenin expression }(P<0.0080) \\
\text { Less likely to exhibit EGFR amplification }(P<0.0051)\end{array}$ & Less commonly express nuclear $\beta$-catenin $(P<0.0080)$ \\
More frequently express CDX2 $(P=0.020)$ & More commonly exhibit EGFR amplification $(P<0.0051)$ \\
Membranous CD10 and cytoplasmic MUC2 in 10\% of neoplastic cells & Neoplasms are negative for MUC2, CDX2, CD10 \\
Negative for MUC5AC and MUC6 & Positive for MUC5AC and MUC6 \\
There were also cases with mixed IHC phenotype and null cases without IHC expression of these markers in both Siewert locations
\end{tabular}

expression was not seen in normal foveolar zone or in foveolar dysplasia; however, Barrett's dysplasia with a goblet cell (intestinalized) component was positive for MUC2. Intestinalized dysplasia also expresses MUC2. In addition, both foveolar dysplasia in the esophagus and gastric adenocarcinomas share similar staining patterns, including loss or aberrant expression of lethal giant larva 2 (LGL2) in foveolar dysplasia, compared to reactive gastric mucosa. In contrast, expression of LGL2 was also frequently lost in areas of IM. ${ }^{35}$

Exploring this dichotomy of gastric versus intestinal lines of differentiation in neoplasms in this region, 157 esophagectomy specimens of BEassociated and GEJ adenocarcinomas were compared, and their anatomic locations classified using the Siewert system. The neoplasms included only distal esophageal and GEJ adenocarcinomas, and the background mucosa was characterized as intestinal versus cardiac type and assessed for dysplasia. Table 1 summarizes comparative immunohistochemical expression profiles of the carcinomas by background mucosa: intestinal versus cardiac type. The Siewert location was significantly associated with the presence or absence of adjacent IM $(P=$ 0.0009). The prevalence of IM was $86 \%$ in Siewert type I and 60\% in Siewert type II adenocarcinomas. CDX2 expression was seen in 53\% of Siewert type I adenocarcinomas and $38 \%$ of Siewert type II adenocarcinomas. Also of note, there were cases that exhibited mixed or null phenotypes. ${ }^{36}$ These findings provide additional evidence that although the majority of EACs and GEJ adenocarcinomas display intestinal-like immunophenotype, cardiac-type differentiation is definitely not limited to or specific for adenocarcinomas arising in the GEJ or stomach, as gastric foveolar dysplasia can be seen in a significant subset of EACs.
Finally, does the neoplastic immunophenotype reflect etiology, such as $H$. pylori infection versus reflux? Seropositivity for Helicobacter is strongly associated with noncardiac gastric adenocarcinoma (adjusted $\mathrm{OR}=7.9$ ) and inversely associated with cardiac gastric adenocarcinoma (adjusted OR = 0.31 ), and $H$. pylori infection is not associated with neoplasia in BE. Therefore, finding $H$. pylori organisms by hematoxylin and eosin (H\&E) or immunohistochemistry suggests an origin of the adenocarcinoma in the stomach, below the GEJ, rather than a GEJ or distal esophageal origin.

In summary, there are trends of immunohistochemical differentiation by location in phenotype of adenocarcinoma; however, that phenotype is not specific for location. The finding of associated $H$. pylori infection favors gastric origin. Other than $H$. pylori detection, immunohistochemical expression patterns of adenocarcinomas around the GEJ appear to be more associated with histological phenotype or lines of differentiation than with anatomical site of origin or location.

\section{How does the pathologist tell from EMR specimens?}

\section{Andrea Grin}

grina@smh.ca

In order to answer this question, we first need to know the normal histology above, at, and just below the GEJ. While the histologic features of the esophagus just above the GEJ and the gastric fundic mucosa just below the GEJ are well established, normal histology at the GEJ is a matter of debate. One line of thinking is that the normal GEJ is composed of a direct transition from squamous to oxyntic mucosa and that any so-called cardiac (formed of mucous cells only) or oxyntocardiac (mixture of parietal and mucous) mucosa is metaplastic 
secondary to damage from GER. This viewpoint is supported by the work of Chandrasoma et al., which includes a prospective autopsy study showing a direct transition from squamous to oxyntic mucosa (with no cardiac mucosa) in the majority of cases. ${ }^{37}$ The opposing view is that the cardia is, in fact, a normal structure but is very short. A pediatric autopsy series (mean age 6.3 years) demonstrated cardiac mucosa (composed of mucous cells only) in all cases with a mean length of $1.8 \mathrm{~mm} .{ }^{38}$ Zhou et al. ${ }^{39}$ found either mucous or mixed mucous/oxyntic mucosa at the GEJ in all fetal and pediatric autopsies examined, with the exception of one case. Similarly, biopsies of the GEJ of pediatric patients (mean age 13.0 years) demonstrated pure mucous glands within $1 \mathrm{~mm}$ of the squamous mucosa in $81 \%$ of patients, and a direct transition from squamous to oxyntic mucosa was not seen. ${ }^{40}$

Regardless of whether a small amount of mucous or mixed mucous/oxyntic glands (i.e., cardia or cardiac-type mucosa) at the GEJ is normal, evidence shows that its length increases with age, acid exposure, and with histologic evidence of esophagitis and carditis. ${ }^{40,41}$ Therefore, metaplastic esophageal mucosa (i.e., BE) is essentially similar to the histology of cardiac mucosa and it can be very difficult, if not impossible, to distinguish these histologically in some cases.

As opposed to biopsies, EMR specimens are larger and ideally include submucosal tissue. These differences are helpful in assessing esophageal location. Features most useful in confirming esophageal location in an EMR specimen are (1) the presence of esophageal submucosal glands and/or ducts, (2) duplicated muscularis mucosae, and (3) squamous islands or buried columnar mucosa with IM. A newly described but less well-appreciated feature, palisade vessels, is also gaining recognition.

\section{Submucosal glands and ducts}

The most reliable feature and one shown to have the highest level of interobserver agreement is the presence of submucosal glands and/or ducts. Submucosal glands are present throughout the length of the esophagus but are more numerous both proximally and distally. Submucosal glands develop during the fourth month of gestation from ciliated columnar mucosa; therefore, their presence can be accepted as histologic evidence of esophageal location.
Submucosal glands and/or ducts can be seen in approximately $20-30 \%$ of EMR specimens. ${ }^{42}$

\section{Duplication of the muscularis mucosae}

Duplication of the muscularis mucosae is clearly associated with $\mathrm{BE}$ and is thought to be part of the morphologic changes of Barrett's metaplasia, which includes not only epithelial metaplasia but also stromal changes. Duplication has not been identified within the stomach nor in esophagectomy specimens without BE. ${ }^{43}$ While a duplicated muscularis mucosa can be identified in approximately $90 \%$ of esophagectomy specimens with $\mathrm{BE},{ }^{7}$ the detection rate is somewhat lower in EMR specimens $(65-70 \%)^{44}$ and the extent of duplication varies and can be focally present. ${ }^{44,45}$ In one study, extensive duplication was seen in $38 \%$ of specimens, moderate in 33\%, and minimal in $29 \%{ }^{44}$ Another EMR study showed minimal $(<10 \%)$ or absent duplication in the majority (54\%) of cases. ${ }^{45}$

\section{Squamous islands or buried columnar mucosa with IM}

Since squamous mucosa is found in the esophagus and not in the stomach, the presence of squamous islands within glandular mucosa and buried columnar mucosa with IM supports an esophageal location. Squamous islands within an area of columnar mucosa have been shown to be a diagnostic feature of BE, and these are often associated with an underlying submucosal gland or duct. ${ }^{46}$ In EMR specimens, squamous islands are seen in approximately $20 \%$ of specimens.

\section{Palisade vessels}

Japanese gastroenterologists use the lower limit of palisade vessels to define the GEJ, but these are not well appreciated histologically. A study by Aida et al. ${ }^{42}$ showed that veins $>100$ um within the mucosa (above the true muscularis mucosae) were specific for esophageal origin in EMR specimens. Venous size within the lower esophagus and within BE was significantly larger than in the mid-esophagus, gastric cardia, and fundus. The authors conclude that palisade vessels are a specific histologic marker of tissue originating from the lower esophagus.

\section{Conclusion}

The normal histology at the GEJ is a matter of debate and the cardia (if it exists) and metaplastic esophageal mucosa show histologic overlap, 
causing difficulty for the pathologist. EMR specimens have the advantage of increased sampling with the inclusion of the submucosa. This may allow for identification of features associated with esophageal location such as submucosal glands/ducts, duplicated muscularis mucosae, and squamous islands/buried columnar mucosa. Palisade vessels are a newly recognized feature. In the absence of one of these features, the pathologist cannot be certain if the EMR is from the esophagus, and in these instances, correlating with endoscopic/clinical impression is required.

\section{Are esophageal pyloric adenomas possible candidates for a precursor lesion of adenocarcinoma arising from cardia? What evidence do we have?}

\author{
Monika Tripathi, Andrea Grin, \\ Catherine J. Streutker, and Michael Vieth \\ monika.tripathi@doctors.org.uk
}

Over the last two decades, with the rising incidence of EAC, there has been a parallel rise in the incidence of GEJ and gastric cardia adenocarcinoma (GCA). ${ }^{47,48}$ In contrast to EAC, the GCAs do not have a strong association with IM nor BE. The possible candidates for precursor lesions for GCAs include IM, H. pylori and carditis, foveolar dysplasia, and gastric-type pyloric adenomas. ${ }^{47}$

Pyloric gland adenoma (PGA) is a rare neoplasm with distinct histomorphology and is frequently associated with transition from dysplasia to carcinoma. PGAs were first classified as a rare gastric tumor in $1990 .{ }^{49}$ They occur predominantly in the elderly and have a slight female predilection. A significant proportion of these are associated with autoimmune gastritis. On histomorphology, they consist of tightly packed pyloric-type glands lined by a single layer of cuboidal to columnar mucussecreting cells containing pale eosinophilic cytoplasm with lack of apical mucin cap, in contrast to gastric foveolar epithelium. They have a characteristic immunophenotype, showing expression of MUC5AC in the deeper parts and MUC6 in the apical surface epithelial layer and are negative for CDX2, CD10, and MUC2. ${ }^{50}$

About $85 \%$ are located in the stomach, predominantly in the fundus (64\%), with a small minority $(7.9 \%)$ within the cardia. ${ }^{51}$ The $15 \%$ of the extragastric sites include gall bladder, pancreatic duct, duodenum, bile duct, uterine cervix, rectum, and two cases within the esophagus. ${ }^{52}$ The first case within the esophagus was reported in by Kushima et al. in $2005 .^{53}$ It was a small $3 \times 2 \mathrm{~mm}$ polyp arising in a background of BE. Immunophenotype was positive for MUC6 and MUC5AC and negative for CD10 and MUC2. Complete genomic hybridization analyses of this lesion showed losses on 2p2425.2, 2q14.1-ter, 5q31.3-32, 6q23-24, 8q23-24.2, 11q22.3-24, and 18q21.1-22. Interestingly, most of the molecular studies on GCA have shown losses of both $5 \mathrm{q}$ and $18 \mathrm{q}$ in more than $50 \%$ of cases, indicating definite association between these two entities. ${ }^{54}$

Following this, one more case of PGA was reported within the lower esophagus; this was a $1.5 \mathrm{~cm}$ bulging tumor growing beneath the squamous epithelium and was associated with conventionaltype low-grade dysplasia, indicating its unstable/ premalignant potential. ${ }^{55}$ But no cases of adenocarcinoma arising in esophageal PGA have yet have been reported in the literature. In contrast, different studies have documented about 30\% transition of gastric PGAs to well-differentiated adenocarcinoma. ${ }^{56}$

Retrospective review of 37 cases of GCAs diagnosed at our center showed only $35.2 \%$ association with background IM and only three cases with $H$. pylori infection, but no case of PGA has been reported at this site. Due to its rarity, there is not enough evidence supporting its malignant transition at the esophageal site (only two cases reported in the indexed literature), although the cases reported so far show definite molecular association between the esophageal PGAs and the GCA. One of the reasons for scant clinical data might be frequent underdiagnosis and unawareness of these lesions at the extragastric sites, and they are likely not as rare as they are described in published literature.

\section{Can pancreatic metaplasia be a possible precursor of adenocarcinoma at the GEJ?}

\section{Sahar Al-Haddad \\ Al-HaddadS@smh.ca}

Metaplasia is the transformation of one type of mature tissue to another type. PAM at the GEJ is the transformation of gastric-type mucosa to pancreatic-type mucosa. It was first reported by 
Doglioni et al. in $1993 .{ }^{57}$ The reported prevalence ranges between $14 \%$ and $24 \% .^{58,59}$

Some authors have argued for a congenital origin for pancreatic metaplasia, whereas others suggested an acquired etiology. Those in favor of an acquired mechanism have shown a relationship between pancreatic metaplasia and GERD and also H. pylori infection in the stomach. ${ }^{60,61}$ One of the most convincing reports of a possible congenital origin for PAM was made in 1999 by Luque-Barona, who studied 67 consecutive infantile autopsies and found two cases of PAM in the stomach, one in a 3-month-old infant who suffered bilateral renal dysplasia and complex polymalformative syndrome and the second in a 5month-old infant with extrahepatic biliary atresia who died from sepsis after surgery. ${ }^{62}$

Johansson et al. found an overall prevalence of PAM of $18 \%$, with $10 \%$ being below the GEJ, $6 \%$ above the GEJ, and $2 \%$ at the level of the GEJ. Those above the GEJ were significantly associated with H. pylori and GERD. ${ }^{61}$

In two studies of a variant of gastric adenocarcinoma with pancreatic acinar-like differentiation arising in the proximal stomach and invading the esophagus, none of the 43 cases in the first study or the case reported in the second study showed pancreatic acinar metaplasia in adjacent benign gastric tissue. ${ }^{63,64}$

$\mathrm{K}$-ras is a tumor oncogene expressed in over $80 \%$ of pancreatic adenocarcinomas. c-erbB2 is a receptor tyrosine kinase implicated in the development of pancreatic, esophageal, and gastric cancers. To study any possible neoplastic potential for PAM, Polkowski et al. investigated the possible expression of c-erbB2 and K-ras in patients with PAM with no evidence of BE, and neither was expressed. ${ }^{60}$

While future studies may produce different findings, the current evidence shows no indication of a neoplastic potential for pancreatic acinar metaplasia.

\section{Is there reason to make a distinction about site of origin with respect to Her2 immunoreactivity?}

Catherine J. Streutker

StreutkerC@smh.ca

The Her2 receptor is a transmembrane receptor protein belonging to the EGFR family. The Her2 protein is involved in a number of cell functions, including growth, differentiation, proliferation, and survival. It is present at low levels on normal gastric epithelial cells, but in a proportion of tumors, it is overexpressed. ${ }^{65}$ The Trastuzumab for Gastric Cancer (ToGA) trial, demonstrating responsiveness of gastric and GEJ adenocarcinomas to traztuzumab, showed significant differences in the rate of Her2 positivity between GEJ (33\%) versus distal stomach $(21 \%)$ adenocarcinomas, most often in moderately to well-differentiated intestinal-type tumors. ${ }^{66}$ Scoring of Her2 for these tumors is significantly different from that used in breast carcinomas (i.e., incomplete membrane staining is allowed and heterogeneity is a frequent finding). ${ }^{67}$ Her2 positivity in several studies was related to aggressive tumors and poor outcome, but this has not been replicated in all studies. ${ }^{68,69}$

Comparing adenocarcinomas of the esophagus to those in the GEJ and stomach has shown some variability. Yoon et al. looked at 713 tumors; $17 \%$ were $\mathrm{Her}^{+}$overall. In EAC, Her2 positivity was $21 \%$, compared to $15 \%$ for those at the GEJ or gastric cardia. ${ }^{70}$ In their study, Her2 positivity correlated with lower tumor grade, less invasiveness, and fewer lymph node metastases. As the presence of Her2-amplified tumors also correlated with the presence of adjacent Barrett's mucosa, the authors concluded that Her2 positivity is strongly associated with the intestinal path of tumor progression at the GEJ, rather than the cardiac type/foveolar dysplasia. In contrast, Phillips et al. found only $15 \%$ of esophageal primary adenocarcinoma and 28\% of GEJ primary adenocarcinoma were $\mathrm{Her} 2^{+}$, and they found that there were no significant differences between the groups in pathology or outcome. ${ }^{71}$

Table 2. The SMH experience of Her2 expression in 658 cases of adenocarcinoma of the distal esophagus, GEJ, cardia, and distal stomach. Overall, $22 \%$ of cases were positive for Her2

\begin{tabular}{lcccc}
\hline Site & $\begin{array}{c}\text { Distal } \\
\text { esophagus }\end{array}$ & GEJ & Cardia & Stomach \\
\hline \# of cases & 79 & 157 & 16 & $\begin{array}{c}406 \text { (approxi- } \\
\text { mately } 40 \% \\
\text { had no site } \\
\text { specified) }\end{array}$ \\
$\begin{array}{c}\text { \% Her2 } \\
\text { \% diffuse/ } \\
\text { mixed }\end{array}$ & $26 \%$ & $35 \%$ & $12.5 \%$ & $18 \%$ \\
\hline
\end{tabular}


St. Michael's Hospital is part of the gastric cancer Her2 testing network for Ontario, Canada. Analysis of tested cases was done to evaluate for the rate of Her2 positivity at, above, and below the GEJ, where noted on the requisitions from incoming cases. (Table 2) This appears to show differences between the different sites; however, this may be entirely due to the rate of diffuse gastric cancer. This type of tumor is rare in the esophagus and is only very rarely positive for Her2 overexpression; the differing rates of Her2 expression between the esophagus, GEJ, and distal stomach may all be due to the variation in the rates of intestinal-type cancers between the sites. Further studies are needed to determine if there are significant biologic differences between these tumors with respect to Her2 positivity and response to trastuzumab.

\section{IM limited to the cardia}

Vincenzo Villanacci and Marianna Salemme villanac@alice.it

The correct definition of the cardiac region is still controversial. In general, it is considered as a carefully localized anatomical region present in healthy individuals, and in this sense Odze wrote "the level at which the esophagus ends and the stomach begins." ${ }^{\prime 2}$ Nevertheless, the experience of Chandrasoma on autopsy ${ }^{37}$ denied this theory. In his autopsy analysis, $30 \%$ of 72 patients did not have any cardiac mucosa, while all 71 patients with reflux disease had cardiac mucosa, suggesting that cardiac mucosa is not a normal structure but is an abnormal mucosa that results from the metaplastic transformation of the squamous epithelium damaged by the reflux. Against this definition, there are the experiences of Kilgore and Glickman: the first, analyzing 30 pediatric autopsies, identified the presence of cardiac mucosa in all cases (ranging from 1 to $4 \mathrm{~mm}$ in length), while the latter studied 74 pediatric patients, in which he identified the diffuse presence of pure mucous glands or mixed mucous/oxyntic glands, typical histological hallmarks of cardiac mucosa. ${ }^{38,40}$ On the basis of these different interpretations, we consider the cardia as a definite area of the stomach that is present both in normal anatomy and in pathological conditions. IM limited to the cardia is a particular histological finding that is detected with variable frequency (ranging from $0 \%$ to $36 \%$ ) by different series, depending on several elements, including endoscopic techniques (e.g., magnification, acetic acid, vital dye), location/number of biopsies, and histological methods (e.g., Alcian blue; Fig. 2). In particular, it is important to highlight the role of a correct biopsy sampling, based on an adequate number of biopsies above (Barrett's area) and below (cardiac region) the Z-line, separating biopsies from these different areas into specific containers. In the literature, several causes of cardiac IM have been studied, such as age, smoking, and overweight ${ }^{73}$ however, most authors identified H. pylori infection and reflux disease as the main factors in the onset of cardiac inflammation and hence of IM (Table 3). ${ }^{74}$ This concept has been also remarked by Goldblum, ${ }^{75}$ according to which the significance of IM near the GEJ in an individual patient should be evaluated in the context of endoscopic findings, histological and serologic data for $H$. pylori infection, and information obtained from biopsies of the distal stomach. The role of IM in the cardiac region has consequently been investigated. In two different series, Cameron et al. ${ }^{76,77}$

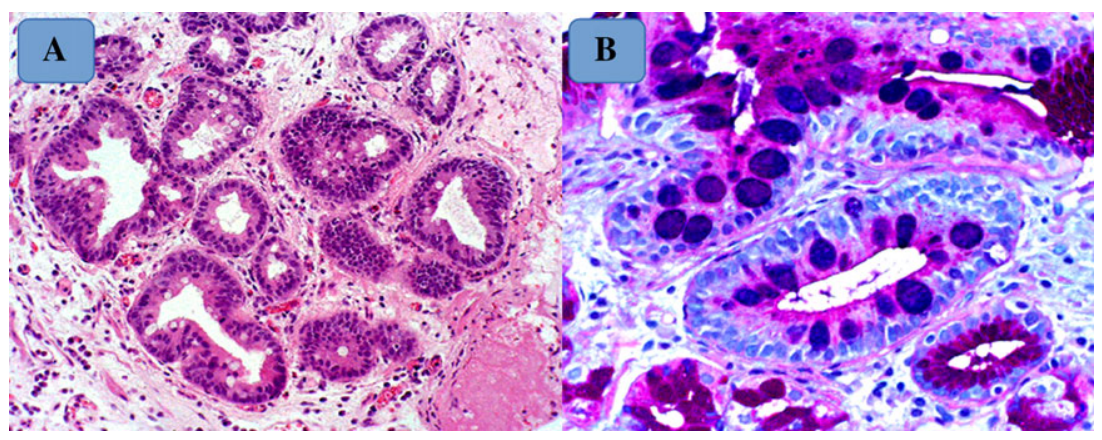

Figure 2. Intestinal metaplasia in the cardiac region. (A) Hematoxylin-eosin, original magnification 10x. (B) Alcian-Pas staining, original magnification $40 \mathrm{x}$. 
Table 3. Concurrent presence of Helicobacter pylori infection/reflux disease at the time of the diagnosis and the development of dysplasia/cancer during the follow-up period

\begin{tabular}{lccccc}
\hline IM in the cardia & $\begin{array}{c}\text { Low-grade } \\
\text { dysplasia }\end{array}$ & $\begin{array}{c}\text { Low- and } \\
\text { high-grade } \\
\text { dysplasia }\end{array}$ & $\begin{array}{c}\text { Intestinal-type } \\
\text { cancer }\end{array}$ & $\begin{array}{c}\text { Diffuse-type } \\
\text { cancer }\end{array}$ & $\begin{array}{c}\text { Mixed-type } \\
\text { cancer }\end{array}$ \\
\hline H. pylori infection & 0 & 0 & 7 & 2 & 2 \\
Reflux disease & 1 & 2 & 12 & 3 & 0 \\
\hline
\end{tabular}

demonstrated that in both small and large adenocarcinomas of the cardia, IM was present with a high frequency (in $86 \%$ and $42 \%$ of cases, respectively), emphasizing the role of IM as an important risk factor for cancer development. In our experience, from 2000 to 2012, we found the histological features of IM limited to the cardia in 133 patients undergoing cardiac biopsies; among these, 30 patients had concurrent $H$. pylori infection, while the remaining cases had a concomitant reflux disease. During the follow-up period, three cases developed dysplasia and 26 developed cancer. The histological analysis of cases with cancer revealed in 19 patients $(73 \%)$ the presence of an intestinal-type cancer. Taken together, the results of our experience emphasize the concept that IM is a frequent lesion in the cardiac region, even when endoscopy is normal and has an important preneoplastic significance in the progression toward dysplasia and cancer.

\section{Conflicts of interest}

The authors declare no conflicts of interest.

\section{References}

1. Siewert, J.R., A.H. Holscher, K. Becker \& W. Gossner. 1987. Kardiacarcinom: Versuch einer therapeutisch relevanten Klassifikation. Chirurg 58: 25-32.

2. Siewert, J.R. \& H.J. Stein. 1998. Classification of adenocarcinoma of the oesophagogastric junction. Br. J. Surg. 85: 1457-1459.

3. Keeney, S. \& T.L. Bauer. 2006. Epidemiology of adenocarcinoma of the esophagogastric junction. Surg. Oncol. Clin. N. Am. 15: 687-696.

4. Feith, M., H.J. Stein \& J.R. Siewert. 2006. Adenocarcinoma of the esophagogastric junction: surgical therapy based on 1602 consecutive resected patients. Surg. Oncol. Clin. N. Am. 15: 751-764.

5. Barbour, A.P., N.P. Rizk, M. Gonen, et al. 2007. Adenocarcinoma of the gastroesophageal junction: influence of esophageal resection margin and operative approach on outcome. Ann. Surg. 246: 1-8.

6. Dresner, S.M., P.J. Lamb, M.K. Bennett, et al. 2001. The pattern of metastatic lymph node dissemination from ade- nocarcinoma of the esophagogastric junction. Surgery 129: 103-109.

7. Marsman, W.A., G.N. Tytgat, F.J. ten Kate \& J.J. van Lanschot. 2005. Differences and similarities of adenocarcinomas of the esophagus and esophagogastric junction. J. Surg. Oncol. 92: 160-168.

8. Rice, T.W., V.W. Rusch, C. Apperson-Hansen, et al. 2009. Worldwide Esophageal Cancer Collaboration. Dis. Esophagus 22: 1-8.

9. Gaur, P., W.L. Hofstetter, B.N. Bekele, et al. 2010. Comparison between established and the Worldwide Esophageal Cancer Collaboration Staging Systems. Ann. Thorac. Surg. 89: 1797-1804, e1793.

10. Rusch, V.W., T.W. Rice, J. Crowley, et al. 2010. The seventh edition of the American Joint Committee on Cancer/International Union Against Cancer Staging Manuals: the new era of data-driven revisions. J. Thorac. Cardiovasc. Surg. 139: 819-821.

11. Wang, H.H., et al. 1986. Comparative features of esophageal and gastric adenocarcinomas: recent changes in type and frequency. Hum. Pathol. 17: 482-487.

12. McColl, K.E. \& J.J. Going. 2010. Aetiology and classification of adenocarcinoma of the gastro-oesophageal junction/cardia. Gut 59: 282-284.

13. Derakhshan, M.H., et al. 2008. Combination of gastric atrophy, reflux symptoms and histological subtype indicates two distinct aetiologies of gastric cardia cancer. Gut 57: 298305.

14. Demicco, E.G., et al. 2011. The dichotomy in carcinogenesis of the distal esophagus and esophagogastric junction: intestinal-type vs cardiac-type mucosaassociated adenocarcinoma. Mod. Pathol. 24: 11771190.

15. Huang, Q., et al. 2011. Comparison of gastro-oesophageal junction carcinomas in Chinese versus American patients. Histopathology 59: 188-197.

16. Srivastava, A., et al. 2007. Morphologic features are useful in distinguishing Barrett esophagus from carditis with intestinal metaplasia. Am. J. Surg. Pathol. 31: 1733-1741.

17. Hansen, S., et al. 2007. Two distinct aetiologies of cardia cancer; evidence from premorbid serological markers of gastric atrophy and Helicobacter pylori status. Gut 56: $918-$ 925.

18. Yantiss, R.K. 2010. Diagnostic challenges in the pathologic evaluation of Barrett esophagus. Arch. Pathol. Lab. Med. 134: 1589-600.

19. Odze, R.D. 2011. What the gastroenterologist needs to know about the histology of Barrett's esophagus. Curr. Opin. Gastroenterol. 27: 389-396. 
20. Becker, K., R. Langer, D. Reim, et al. 2011. Significance of histopathological tumor regression after neoadjuvant chemotherapy in gastric adenocarcinomas: a summary of 480 cases. Ann. Surg. 253: 934-939.

21. Chirieac, L.R., S.G. Swisher, J.A. Ajani, et al. 2005. Posttherapy pathologic stage predicts survival in patients with esophageal carcinoma receiving preoperative chemoradiation. Cancer 103: 1347-1355.

22. Langer, R., K. Ott, M. Feith, et al. 2009. Prognostic significance of histopathological tumor regression after neoadjuvant chemotherapy in esophageal adenocarcinomas. Mod. Pathol. 22: 1555-1563.

23. Reim, D., R. Gertler, A. Novotny, et al. 2012. Adenocarcinomas of the esophagogastric junction are more likely to respond to preoperative chemotherapy than distal gastric cancer. Ann. Surg. Oncol. 19: 2108-2118.

24. Thies, S. \& R. Langer. 2013. Tumor regression grading of gastrointestinal carcinomas after neoadjuvant treatment. Front Oncol. 3: 262.

25. Theisen, J., H.J. Stein \& H.J. Dittler. 2002. Preoperative chemotherapy unmasks underlying Barrett's mucosa in patients with adenocarcinoma of the distal esophagus. Surg. Endosc. 16: 671-673.

26. Bosman, F.T., F. Carneiro, R.H. Hruban \& N.D. Theise, Eds. 2010. WHO Classification of Tumours of the Digestive System. Lyon: World Health Organization.

27. Edge, S.B., D.R. Byrd, C.C. Compton, et al. 2009. AJCC Cancer Staging Manual. Berlin: Springer.

28. Sobin, L.H., M.K. Gospodarowicz \& Ch. Wittekind, Eds. 2009. TNM Classification of Malignant Tumours, 7th Ed. New York: Wiley-Blackwell.

29. Kalish, R.J., P.E. Clancy, M.B. Orringer \& H.D. Appelman. 1984. Clinical, epidemiologic, and morphologic comparison between adenocarcinomas arising in barretts esophageal mucosa and in the gastric cardia. Gastroenterology 86: 461467.

30. Heidl, G., P. Langhans, W. Mellin, et al.1993. Adenocarcinomas of esophagus and cardia in comparison with gastriccarcinoma. J. Cancer Res. Clin. Oncol. 120: 95-99.

31. Sarbia, M. 2006. The histological appearance of oesophageal adenocarcinoma-an analysis based on 215 resection specimens. Virchows. Archiv. 448: 532-538.

32. Amano, Y., N. Ishimura, K. Furuta, et al. 2006. Which landmark results in a more consistent diagnosis of Barrett's esophagus, the gastric folds or the palisade vessels? Gastrointest. Endosc. 64: 206-211.

33. Senoo, M., F. Pinta \& C.P. Crum. 2007. p63 is essential for the proliferative potential of stem cells in stratified epithelia. Cell 129: 523-569.

34. Wang, X., H. Ouyang, Y. Yamamoto, et al. 2011. Residual embryonic cells as precursors of Barrett's-like metaplasia. Cell 145: 1023-1035.

35. Patil, D.T., A. Bennett, D. Mahaja, et al. 2013. Distinguishing Barrett gastric foveolar dysplasia from reactive cardiac mucosa in gastroesophageal reflux disease. Hum. Pathol. 44: 1146-1153.

36. Xian, Y., K.Y. Ho, C.P. Crum. 2012. Cellular origin of Barrett's esophagus: controversy and therapeutic implications. Gastroenterology 142: 1424-1430.
37. Chandrasoma, P.T. et al. 2000. Histology of the gastroesophageal junction: an autopsy study. Am. J. Surg. Pathol. 24: 402-409.

38. Kilgore, S.P. et al. 2000. The gastric cardia: fact or fiction? Am. J. Gastroenterol. 95: 921-924.

39. Zhou, H. et al. 2001. Origin of cardiac mucosa: ontogenic consideration. Pediatr. Dev. Pathol. 4: 358-363.

40. Glickman, J.N. et al. 2002. Morphology of the cardia and significance of carditis in pediatric patients. Am. J. Surg. Pathol. 26: 1032-1039.

41. Chandrasoma, P.T. et al. 2000. Definition of histopathologic changes in gastroesophageal reflux disease. Am. J. Surg. Pathol. 24: 344-351.

42. Aida, J. et al. 2011. Palisade vessels as a new histologic marker of esophageal origin in ER specimens from columnar-lined esophagus. Am. J. Surg. Pathol. 35: 1140-1145.

43. Takubo, K. et al. 1991. Double muscularis mucosae in Barrett's esophagus. Hum. Pathol. 22: 1158-1161.

44. Lewis, J.T., K.K. Wang \& S.C. Abraham. 2008. Muscularis mucosae duplication and the musculo-fibrous anomaly in endoscopic mucosal resections for barrett esophagus: implications for staging of adenocarcinoma. Am. J. Surg. Pathol. 32: 566-571.

45. Mandal, R.V. et al. 2009. Effect of tumor characteristics and duplication of the muscularis mucosae on the endoscopic staging of superficial Barrett esophagus-related neoplasia. Am. J. Surg. Pathol. 33: 620-625.

46. Takubo, K. et al. 2005. Islands of squamous epithelium and their surrounding mucosa in columnar-lined esophagus: a pathognomonic feature of Barrett's esophagus? Hum. Pathol. 36: $269-274$.

47. Jovanovic, I. \& Y.A. Mouzas. 2001. Gastric cardia cancer and precursor lesions- current dilemmas. Ann. Gastroenterol. 14: 244-251.

48. Kushima, R., M. Vieth, F. Borchard, et al. 2006. Gastric type well-differentiated adenocarcinoma and pyloric gland adenoma of the stomach. Gastric Cancer 9: 177184.

49. Borchard, F., A. Ghanei, U. Koldovsky, et al. 1990. Gastrale Differenzierung in Adenomen der Magenschleimhaut. Immunohistochemische und elektronenmikroskopische Untersuchungen. Verh Dtsch. Ges. Pathol. 74: 528.

50. Vieth, M., C. Vogel, R. Kushima, et al. 2006. Pyloric gland adenoma: how to diagnose? Cesk. Patol. 42: 4-7.

51. Vieth, M., R. Kushima, F. Borchard, et al. 2003. Pyloric gland adenoma: a clinico-pathological analysis of 90 cases. Virchows. Arch. 442: 317-321.

52. Vieth, M., R. Kushima, J.P. de Jonge, et al. 2005. Adenoma with gastric differentiation (so-called pyloric gland adenoma) in a heterotopic gastric corpus mucosa in the rectum. Virchows. Arch. 446: 542-545.

53. Kushima, R., M. Vieth, K. Mukaisho, et al. 2005. Pyloric gland adenoma arising in Barrett's esophagus with mucin immunohistochemical and molecular cytogenetic evaluation. Virchows. Arch. 446: 537-541.

54. Buffart, T.E., B. Carvalho, T. Mons, et al. 2007. DNA copy number profiles of gastric cancer precursor lesions. $B M C$ Genomics 8: 345 . 
55. Michal, M., R. Curik, K. Matler, et al. 2003. Regarding the paper by Vieth et al. Virchows Archiv. 442/4: 317-321. Virchows Arch. 443: 589-590.

56. Ahn, J.Y., S.O. Suh, J.O. Jung, et al. 2011. A case of pyloric gland adenoma in the cardia of the stomach, treated by endoscopic submucosal dissection. Korean J. Med. 80: 7881.

57. Doglioni, C., L. Laurino, A.P. Dei Tos, et al. 1993. Pancreatic (acinar) metaplasia of the gastric mucosa. Histology, ultrastructure, immunocytochemistry, and clinicopathologic correlations of 101 cases. Am. J. Surg. Pathol. 17: 1134-1143.

58. Wang, H.H., J.M. Zeroogian, S.J. Spechler, et al. 1996. Prevalence and significance of pancreatic acinar metaplasia at the gastroesophageal junction. Am. J. Surg. Pathol. 20: 15071510 .

59. Johansson, J., H.O. Hakansson, L. Mellblom, et al. 2005. Prevalence of precancerous and other metaplasia in the distal oesophagus and gastro-oesophageal junction. Scand. J. Gastroenterol. 40: 893-902.

60. Polkowski, W., J.J. van Lanschot, F.J. ten Kate, et al. 2000. Intestinal and pancreatic metaplasia at the esophagogastric junction in patients without Barrett's esophagus. Am. J. Gastroenterol. 95: 617-625.

61. Johansson, J., H.O. Hakansson, L. Mellblom, et al. 2010. Pancreatic acinar metaplasia in the distal oesophagus and the gastric cardia: prevalence, predictors and relation to GORD. J. Gastroenterol. 45: 291-299.

62. Luque-Barona, R.J., T. Pereda-Salguero \& J. FernandezAlonso. 1999. So-called pancreatic acinar metaplasia of the gastric mucosa in two infants: a post-mortem study. Eur. J. Pediatr. 158: 267-268.

63. Huang, Q., J.S. Gold, J. Shi, et al. 2012. Pancreatic acinarlike adenocarcinoma of the proximal stomach invading the esophagus. Hum. Pathol. 43: 911-920.

64. Lee, H., L.H. Tang, E.F. Veras \& D.S. Klimstra. 2012. The prevalence of pancreatic acinar differentiation in gastric adenocarcinoma: report of a case and immunohistochemical study of 111 additional cases. Am. J. Surg. Pathol. 36: 402408.

65. Bang, Y.J. 2012. Advances in the management of HER2positive advanced gastric and gastroesophageal junction cancer. J. Clin. Gastroenterol. 46: 637-648.

66. Bang, Y.J., E. Van Cutsem, A. Feyereislova, et al. 2010. Trastuzumab in combination with chemotherapy versus chemotherapy alone for treatment of HER2-positive advanced gastric or gastroesophageal junction cancer (ToGA): a phase 3 open-label randomized controlled trial. Lancet 376: 687-697.

67. Rüschoff, J., W. Hanna, M. Bilous, et al. 2012. HER2 testing in gastric cancer: a practical approach. Mod Pathol. 25: 637650.

68. Begnami, M.D., E. Fukuda, J.H. Fregnani, et al. 2011. Prognostic implications of altered human epidermal growth factor receptors (HERs) in gastric carcinomas: HER2 and HER3 are predictors of poor outcome. J. Clin. Oncol. 29: 30303036.

69. Maresch, J., S.F. Schoppmann, C.M. Thallinger, et al. 2012. Her-2/neu gene amplification and over-expression in stomach and esophageal adenocarcinoma: from pathology to treatment. Crit. Rev. Oncol. Hematol. 82: 310322.

70. Yoon, H.H., Q. Shi, W.R. Sukov, et al. 2012. Association of HER2/ErbB2 expression and gene amplification with pathologic features and prognosis in esophageal adenocarcinomas. Clin. Cancer Res. 18: 546-554.

71. Phillips, B.E., R.R. Tubbs, T.W. Rice, et al. 2013. Clinicopathologic features and treatment outcomes of patient with human epidermal growth factor receptor 2-positive adenocarcinoma of the esophagus and gastroesophageal junction. Dis. Esophagus 26: 299-304.

72. Odze, R.D. 2005. Unraveling the mystery of the gastroesophageal junction: a pathologist's perspective. Am. J. Gastroenterol. 100: 1853-1867.

73. Felley, C., H. Bouzourene, M.B. VanMelle, et al. 2012. Age, smoking and overweight contribute to the development of intestinal metaplasia of the cardia. World J. Gastroenterol. 18: 2076-2083.

74. Cestari, R., V. Villanacci, G. Bassotti, et al. 2007. The pathology of gastric cardia: a prospective, endoscopic, and morphologic study. Am. J. Surg. Pathol. 31: 706-710.

75. Goldblum, J.R. 2003. Barrett's esophagus and Barrett'srelated dysplasia. Mod Pathol. 16: 316-324.

76. Cameron, A.J., E.O. Souto, T.C. Smyrk. 2002. Small adenocarcinomas of the esophagogastric junction: association with intestinal metaplasia and dysplasia. Am. J. Gastroenterol. 97: 1375-1380.

77. Cameron, A.J. 1994. Epidemiology of Barrett's esophagus. Gastroenterol. Clin. Biol. 18(1 Pt 2): D3-D4. 